\title{
Revisão sistemática sobre avaliação psicológica nas pesquisas em equoterapia'
}

\author{
Renata de Souza Zamo ${ }^{2}$ \\ Clarissa Marceli Trentini \\ Universidade Federal do Rio Grande do Sul, Porto-Alegre - RS - Brasil
}

\begin{abstract}
Resumo: $O$ presente artigo teve como objetivo investigar as pesquisas empíricas em equoterapia publicadas entre 2004 e 20I4. Buscamos verificar características das amostras, construtos psicológicos avaliados, instrumentos de avaliação utilizados e resultados psicológicos atribuídos ao tratamento de equoterapia. Foram analisados 20 estudos empíricos, e a grande maioria deles foi realizado com crianças e adolescentes, seis estudos foram realizados com pessoas com Transtorno do Espectro do Autismo. As técnicas de mensuração utilizadas foram técnicas de entrevistas, observação do comportamento, questionários, escalas de qualidade de vida, de funcionalidade e para grupos específicos. Os resultados mais evidentes da equoterapia no âmbito psicológico envolveram a diminuição da ansiedade e os ganhos em relacionamento social. Os ganhos em aspectos cognitivos não ficaram claros. Comparando os estudos brasileiros e internacionais, a necessidade de sistematização da busca por medidas mais fidedignas ocorre em ambos, porém no Brasil parece não haver uma cultura de psicologia baseada em evidências na equoterapia.
\end{abstract}

Palavras-chave: psicologia; equoterapia; revisão sistemática; avaliação psicológica; terapia assistida por cavalos.

\section{PSYCHOLOGICAL ASSESSMENT AND THERAPEUTIC RIDING: A SYSTEMATIC} REVIEW

\begin{abstract}
This paper aims at investigating empirical researches on therapeutic riding published between 2004 and 2014. We seek to verify characteristics of the samples, psychological constructs assessed, assessment tools, and psychological effects attributed to the processing of therapeutic riding. We analyzed twenty empirical studies, the vast majority of them were held with children and adolescents, six studies were done with people with Autism Spectrum Disorder. The measurement techniques mostly used were: interviewing techniques; behavioral observation; questionnaires; quality of life scales; functionality scales and specific groups scales. The most obvious results of therapeutic riding in the psychological context involved decreased anxiety and gains in social relationship. Gains in cognitive aspects were not clear. Comparing Brazilian and international studies, we have a lack of control group and of reliable measurements in both cases, but Brazil, does not seem to have a psychology culture in use research based on evidence in therapeutic riding.
\end{abstract}

Keywords: psychology; therapeutic riding; systematic review; pychological evaluation; equine-assisted therapy.

${ }^{1}$ Agradecimentos ao Conselho Nacional de Desenvolvimento Científico e Tecnológico (CNPq) pelo auxílio ao projeto de pesquisa e à Comissão de Aperfeiçoamento de Pessoal do Nível Superior (Capes), pela bolsa de doutorado.

${ }^{2}$ Endereço para correspondência: Rua Irmão Norberto Francisco Rouch, 755, ap. 616, Jardim Carvalho - Porto Alegre-RS. CEP: 91450-147. 
EVALUACIÓN PSICOLÓGICA Y EQUITACIÓN TERAPÉUTICA: UNA REVISIÓN SISTEMÁTICA

\begin{abstract}
Resumen: Este artículo tiene como objetivo investigar estudios sobre equitación terapéutica publicados entre 2004 y 20I4. Buscamos características de las muestras, constructos psicológicos evaluados, instrumentos de evaluación utilizados y resultados psicológicos atribuibles al tratamiento de equitación terapéutica. Se analizaron 20 estudios empíricos, la mayoría fueron llevados a cabo con niños y adolescentes, seis estudios con personas con Trastorno del Espectro Autista. Las técnicas de medición utilizadas fueron las técnicas de entrevista, la observación del comportamiento, cuestionarios, escalas de calidad de vida, la función y para grupos específicos. Los resultados más evidentes de la equitación terapéutica en el contexto psicológico fueron disminución de la ansiedad y las ganancias en las redes sociales. Las ganancias en los aspectos cognitivos no eran claras. Comparándose los estudios brasileros e internacionales hay necesidad de sistematizar la búsqueda de medidas más fiables en ambos, pero en Brasil no parece haber la cultura de estudios basados en la evidencia.
\end{abstract}

Palabras clave: psicología; equitación terapéutica; revisión sistemática; evaluación psicológica; terapía assistida por cavallos.

\title{
Introdução
}

Nas últimas décadas, várias modalidades de tratamento mediados por animais têm surgido, entre elas, a equoterapia. O marco fundamental da equoterapia brasileira foi a fundação da Associação Nacional de Equoterapia (Ande/BRASIL), em 1989. Esta é responsável pela formação de profissionais e regulamentação dos centros de equoterapia (Associação Nacional de Equoterapia, 2016). Portanto, esse é um método de tratamento recente no Brasil e se destina a atender pessoas deficientes por meio de uma perspectiva biopsicossocial. Segundo a Ande/BRASIL, a Equoterapia seria um método complementar que utiliza o cavalo como facilitador e mediador dos processos terapêuticos e educacionais, de reabilitação e socialização. A terapia é realizada de forma interdisciplinar nas áreas de saúde e educação, utilizando os fundamentos da equitação (Associação Nacional de Equoterapia, 2016).

Algumas mudanças nas técnicas terapêuticas refletem uma busca por perspectivas mais amplas sobre o uso do cavalo com fins terapêuticos o que gerou alterações nas nomenclaturas de associações. Além de ser um instrumento cinesioterapêutico, o cavalo é um ser em interação recíproca, por isso as mudanças ocorrem. O cavalo, quando utilizado por profissionais da saúde mental credenciados/qualificados, pode proporcionar a reabilitação de funções mentais do praticante. A Federação Internacional de Equoterapia - Federation Riding Disabled International (FRDI), fundada em 1980, hoje se chama Federation of Horses in Education and Therapy International (AISBL). A North American Riding for the Handicapped Association (NAHA), Associação Norte-Americana de Equitação para Deficientes também mudou seu nome, e atualmente se chama Professional Association of Therapeutic Horsemanship International (PATH) (PATH, 2016). Dessa forma, acompanhando as mudanças na perspectiva desta área, estudos sobre a eficácia terapêutica desse método para além dos benefícios físicos também devem ser realizados.

A equoterapia no Brasil foi regulamentada utilizando os fundamentos da equitação nas áreas de saúde e de educação. Os fundadores da Ande/BRASIL criaram o termo 
"equoterapia", uma palavra nova, a fim de registrá-la no Brasil. Para isso, basearam sua construção no latim, origem da língua portuguesa, através do radical Equo, derivado do Equus (cavalo), e optaram por homenagear Hipócrates, referência na medicina ocidental, que utilizava a palavra Therapeia para denominar a aplicação do conhecimento técnico-científico em reabilitação (Associação Nacional de Equoterapia, 2016).

Nesta abordagem, o sujeito é responsável pelo seu processo terapêutico e o cavalo é um facilitador, porque possibilita o desenvolvimento nas áreas física, psicológica, educacional e social. A nomenclatura utilizada para quem é atendido em equoterapia é praticante, visto que tal abordagem o considera um agente de sua reabilitação e educação/reeducação na medida em que ele interage com o cavalo (Associação Nacional de Equoterapia, 2016).

Há quatro programas de atendimento em equoterapia reconhecidos no Brasil, são eles: hipoterapia, educação/reeducação, pré-esportivo e prática esportiva paraequestre. Em hipoterapia, o animal atua principalmente como instrumento cinesioterapêutico. Nesse programa é necessário um auxiliar-guia para conduzir o cavalo, porque o praticante não apresenta condições de guiá-lo ou de manter-se sozinho sobre ele. Os demais programas da equoterapia se diferenciam porque os objetivos terapêuticos são distintos e o grau de autonomia do praticante aumenta gradativamente. Na educação/reeducação o enfoque são aspectos psicológicos e educativos e nos pré-esportivo e esportivo há ênfase na atuação do instrutor de equitação com as diferentes modalidades de esportes equestres (Associação Nacional de Equoterapia, 2016). Porém não existe um guideline ou testagens que façam tal ponto de corte e definam o que seria um grau adequado de autonomia sobre o cavalo. O programa de hipoterapia (hippotherapy) consiste na atuação mais veemente de fisioterapeutas, fonoaudiólogos e terapeutas ocupacionais (Smith-Osborne, \& Selby, 2010).

No Brasil, o papel do psicólogo em todos os programas, independentemente do foco, tem sido valorizado (Cruz, 2010). A equoterapia visa organizar as funções cognitivas mais complexas, como atenção, memória e linguagem, por meio nível sensorial estimulado pelo movimento do cavalo, pelo contato com o animal, sua linguagem não verbal e docilidade, a fim de estabelecer com quem monta uma relação afetiva na qual as possibilidades de desenvolvimento pessoal crescem. Medeiros e Dias (2002) consideram o manuseio do cavalo (alimentação e limpeza) fundamental para o desenvolvimento da terapia. Um dos objetivos da equoterapia é auxiliar na aquisição e no desenvolvimento das funções psicomotoras em pessoas com transtornos de humor, da atenção e de ansiedade (Schultz, Remick-Barlow, \& Robbins, 2007). Além disso, a atividade exige do cavaleiro o planejamento e a criação de estratégias a fim de superar os obstáculos impostos pelo próprio cavalo ou desafios e tarefas propostos pela equipe interdisciplinar durante as intervenções (Medeiros, \& Dias, 2002). Desta perspectiva, pode ocorrer a atuação do psicólogo.

Podemos inferir que a psicomotricidade é desenvolvida quando o cavalo se desloca ao passo. Essa andadura impõe ao praticante um movimento ritmado, simétrico e re- 
petitivo que exige do cavaleiro a capacidade de adaptar-se ao tempo de repouso e de atividade que incide alternadamente a fim de manter-se equilibrado (Malta, 2002). Assim, o praticante é conduzido a compreender melhor seu esquema corporal por meio de suas atitudes corporais de reajuste de postura. A compreensão verbal e o esquema corporal também podem ser avaliados e estimulados pela nomeação e comparação das partes do corpo do sujeito e do cavalo (Malta, 2002).

A priori, a estimulação do sistema nervoso central se dá, à medida que o cavalo anda ao passo, por vias nervosas aferentes. O movimento tridimensional transmitido ao cavaleiro estimula que haja ajuste tônico, melhora do equilíbrio, alinhamento corporal, consciência corporal, coordenação motora e força muscular em resposta aos estímulos recebidos (Giagazoglou, Arabatzi, Dipla, Liga, \& Kellis, 2012; Shurtleff, Standeven, \& Engsberg, 2009). Através do alinhamento gravitacional da díade homem-cavalo, um em relação ao outro se encontra imóvel, porém, em relação ao solo está em movimento (Medeiros \& Dias, 2002).

Freire (1999) refere dados de palestra proferida por Citterio em 1991, que mostrou dados de pesquisa realizada na Itália cujos ganhos neuropsicológicos em sustentação da atenção, melhora em orientação e organização espacial, além de maior capacidade executiva, são atribuídos ao tratamento de equoterapia. Além disto, sugeriu melhora na "capacidade de expressão, de execução e melhor canalização da agressividade" (Freire, 1999, p. 34).

A grande maioria dos estudos em equoterapia cita como objetivo da reabilitação a funcionalidade motora e geralmente visa mensurar a aquisição de benefícios físicos como equilíbrio e força em pessoas com paralisia cerebral (Giagazoglou et al., 2012; Shurtleff et al., 2009). Talvez a escassez de estudos empíricos sobre os componentes emocionais e cognitivos envolvidos no tratamento de equoterapia seja um reflexo das limitações da pesquisa na área clínica. Desde o início deste século, pesquisadores buscam sistematizar estudos nesta área, como Pauw (2000), que aponta os problemas metodológicos nas pesquisas em equoterapia. Tal estudo aponta problemas e desafios encontrados pelos pesquisadores da área, como: uso de diferentes instrumentos de medida; ou diferentes técnicas estatísticas; tamanho amostral pequeno; falta de grupo controle; pouco controle das variáveis intervenientes etc. Esse estudo também aponta a dificuldade em diferenciar o que seriam os benefícios físicos dos psicológicos, afinal, nas análises quantitativas, não se verificam resultados estatisticamente significantes, porém as análises qualitativas de entrevistas a familiares e terapeutas apontam benefícios ao praticante depois do início da equoterapia. Há uma distinção entre estatisticamente significativo e clinicamente significativo, conforme foi salientado nesse artigo (Pauw, 2000).

Estudos sobre a eficácia terapêutica de métodos como a equoterapia são importantes para os profissionais, pacientes e instituições que promovem e financiam esta modalidade de tratamento com o uso do cavalo. No Brasil, há um projeto de lei, PL-4761/2012, regulamentando a equoterapia como um dos recursos a serem disponibilizados à popu- 
lação pelo Sistema Único de Saúde (SUS) (Câmara dos Deputados, 2016). Em 6 de abril de 2016, o parecer de regulamentação da equoterapia foi aprovado pela Comissão de Direitos Humanos e atualmente aguarda a designação do relator da Comissão de Assuntos Sociais do Senado Federal (Senado Federal, 2016).

Este estudo propôs levantar como os construtos psicológicos em equoterapia são mensurados e se há referência de benefícios desta em estudos científicos. Visamos, como objetivo geral, investigar quais construtos psicológicos são avaliados nas pesquisas empíricas em equoterapia. Além disso, buscamos observar a qual população se destinam e como tais construtos são mensurados, ou seja, quais instrumentos de avaliação, sejam eles escalas da área da saúde, sejam testes de uso exclusivo dos psicólogos, foram utilizados em publicações sobre equoterapia. Por conseguinte, verificamos quais foram os principais desfechos apontados pelos estudos empíricos publicados em periódicos revisados por pares.

\section{Método}

Este é um estudo de revisão sistemática de pesquisas empíricas a partir dos seguintes descritores: therapeutic riding, equine assisted learning, equine assisted therapy, equine facilitated mental health, equine assisted psychotherapy, equinoterapia, Equoterapia, terapia assistida por animais. As bases de dados foram: Academic Search Premier (EBSCO), Cambridge Journals Online, JSTOR Arts \& Sciences, Oxford Journals (Oxford University Press), Project Muse, SpringerLink (MetaPress), Wiley Online Library, Scielo, BVSPsi, PubMed, Science Direct, Health Advance e Elsevier Health.

Os estudos incluídos na revisão foram publicados em periódicos revisados por pares de 2004 até 2014, escritos em inglês, português, espanhol e francês. Analisamos somente estudos empíricos, randomizados ou não e sem restrições quanto à análise de avaliadores-cego. Excluímos da pesquisa estudos que enfocaram exclusivamente os benefícios físicos como equilíbrio, postura e amplitude de movimento. Fizeram parte deste estudo pesquisas sobre aspectos psicomotores, funcionalidade e atividades de vida diária, cognição, emoção e outras funções mentais (linguagem, atenção etc.). Consideramos estudos com questões psicológicas aqueles que buscaram verificar atribuições relacionadas à atuação do psicólogo. São atuações do psicólogo estudar, avaliar e intervir no desenvolvimento emocional e de processos mentais e sociais individuais ou coletivos. Dentre as atribuições de sua atuação estão: a promoção de desenvolvimento interpessoal e de integração psíquica e corporal, a mediação de conflitos, e a reabilitação comportamental e cognitiva, dentre outras (Ministério do Trabalho e Emprego, 2016).

\section{Análise dos dados}

Os estudos foram categorizados em empíricos ou teóricos e físico ou psicológicos, segundo análise de dois avaliadores por meio da leitura dos resumos ou na íntegra dos 
artigos. Os avaliadores cruzaram os dados sobre os estudos e, quando houve discordância, um terceiro juiz foi recrutado para decidir se a pesquisa seria incluída ou não no estudo de revisão sistemática. Posteriormente, os estudos que preencheram os critérios de inclusão dessa revisão foram analisados segundo as seguintes categorias: participantes, instrumento ou técnica de avaliação e principais resultados.

\section{Resultados}

Foram levantados 269 artigos, desses foram excluídos os duplicados, estudos com ênfase em questões veterinárias, ou benefícios físicos da equoterapia, e incluídos artigos do acervo pessoal dos autores que cumpriam os critérios de inclusão do estudo. Ao final, analisamos 20 artigos. O Quadro 1 apresenta os participantes, os instrumentos utilizados e os principais desfechos das pesquisas em equoterapia que mostram o predomínio de investigações dos aspectos psicológicos, ou seja, emocionais, sociais ou cognitivos.

Entre os 20 artigos analisados, somente cinco estudos foram conduzidos com sujeitos adultos; um deles não especificou qual diagnóstico os participantes apresentavam (Klontz, Bivens, Leinart, \& Klontz, 2007); um foi realizado com mulheres diagnosticadas com TAG (Motti, \& Freire, 2008); um com grupo com esquizofrenia (Cerino, Cirulli, Chiarotti, \& Seripa, 2011); e dois estudos com pacientes neurológicos (esclerose múltipla, AVC etc.) (Favali \& Milton, 2010; Sunwoo et al., 2012). A maioria dos estudos foi realizada com crianças e adolescentes. Seis pesquisas foram feitas com grupos clínicos com TEA (Gabriels et al., 2012, Hameury et al., 2010; Jenkins \& Reed 2013; Keino et al., 2009; Lanning, Baiter, Ivey-Hatz, Krenek, \& Tubbs, 2014; Memisevikj \& Hodzhikj, 2010), quatro com problemas emocionais, transtorno de aprendizagem ou com queixas de comportamento externalizantes e disruptivos e outros transtornos mentais (Bachi, Terkel, \& Teichman, 2012; Holmes, Goodwin, Redhead, \& Goymour, 2012; Prestes, Weiss, \& Araújo, 2010; Shultz et al., 2007).

Há estudos que, embora englobem questões físicas, se detiveram em avaliar aspectos emocionais, cognitivos ou sociais. Esses foram realizados com participantes com as seguintes características: atraso do desenvolvimento, paralisia cerebral ou com deficiências não especificadas (Ávila, Brito, Pupo, \& Roselló, 2006; Davis et al., 2009; Marcelino, \& Melo, 2006; Rosario-Montejo, Molina-Rueda, Muñoz-Lasa, \& Aguacil-Diego, 2014) e com pessoas com cegueira congênita (Silva \& Grubits, 2004).

Os métodos escolhidos pelos pesquisadores para avaliar os efeitos da equoterapia foram longitudinal com etapa pré e pós-intervenção e grupos comparativos com grupo controle ou intersujeitos. Os estudos brasileiros, em sua maioria, eram qualitativos sem grupo controle (Marcelino \& Melo, 2006; Motti \& Freire, 2008; Silva \& Grubits, 2004). A maior parte dos estudos analisados eram quantitativos e utilizaram instrumentos e escalas validadas, padronizadas e normatizadas para suas populações. Uma das limitações citadas pela maioria dos estudos foi a heterogeneidade dos participantes e a existência de terapias complementares à equoterapia, essas variáveis intervenientes não puderam ser evitadas por questões éticas. 
Os resultados mais evidentes da equoterapia no âmbito psicológico envolveram diminuição da ansiedade (Holmes et al., 2012; Keino et al., 2009; Motti \& Freire, 2008), ganhos em relacionamento social (comunicação, atenção e regras sociais) (Ávila et al., 2006; Cerino et al., 2011; Favali \& Milton, 2010; Gabriels et al., 2012; Hameury et al., 2010; Jenkins, \& Reed, 2013; Keino et al., 2009; Lanning et al., 2014; Memisevikj, \& Hodzhikj, 2010; Silva, 2003; Silva \& Grubits, 2004). Os ganhos em aspectos cognitivos (aprendizagem e outras funções relacionadas) não ficam claros, embora alguns estudos os citem (Ávila et al., 2006; Memisevikj \& Hodzhikj, 2010; Silva, \& Grubits, 2004).

Sobre o uso de instrumentos de medida, verificamos que, ainda que no Brasil tenhamos mais de 150 testes psicológicos validados, padronizados e normatizados para nossa população (http://satepsi.cfp.org.br/listaTeste.cfm?status=1), a grande maioria das pesquisas brasileiras revisadas utiliza entrevistas e observação de comportamentos (Marcelino \& Melo, 2006; Motti \& Freire, 2008; Silva \& Grubits, 2004). Portanto, não fazemos uso dessas ferramentas no momento da avaliação de eficácia terapêutica da equoterapia. Esses dados vão ao encontro do estudo de levantamento com profissionais da equoterapia no Brasil, que dentre os participantes somente um reportou o uso de instrumentos exclusivos do psicólogo como do desenho da figura humana (DFH III) e do desenho da casa, árvore e pessoa (HTP) (Zamo, 2016). Em nossa revisão, somente um estudo utilizou uma escala que está em processo de validação, a Escala do Perfil de Auto- percepção para Crianças (EPAPC) adaptada para o Brasil (Prestes et al., 2010).

No entanto, os estudos internacionais utilizaram questionários, escalas de qualidade de vida, de funcionalidade e para grupos específicos como: Global Assessment of Functioning (GAF), Brief Symptom Inventory (BSI), Personal Orientation Inventory (POI), Child Health Questionnaire, Cerebral Palsy QoL-child Kidscreen, Escala Human-Equips-Interaction on Mental Activity Scale (HEIM), Chelle d'Évaluation des Comportements Autistiques (ECA), Grille d'Évaluation Fonctionnelle des Comportements (EFC), Autism Treatment Evaluation Checklist (ATEC), Brief Psychiatric Rating Scale (BPRS) e 8-Items Positive and Negative Syndrome Scale (PANSS), Spence Children's Anxiety Scale (SCAS), Rosenberg Self-Esteem Scale (SES), Hamilton Depression Rating Scale (Ham-D) e Korean Beck Depression Inventory (K-BDI), dentre outros. Para tanto, podemos inferir que o pouco uso de escalas e instrumentos estruturados em psicologia para avaliar faz parte da falta de hábito do brasileiro em utilizar essas ferramentas, ou da falha na formação nesta área da psicologia que vem sendo discutida (Nunes et al., 2012). 


\section{Quadro I. Pesquisas recentes em equoterapia}

\begin{tabular}{|c|c|c|c|}
\hline Autor/ano & Participantes & Instrumentos & Resultados \\
\hline $\begin{array}{l}\text { Silva \& Grubits } \\
(2004)\end{array}$ & $\begin{array}{l}\text { Cinco crianças (de } 5 \\
\text { a II anos) com } \\
\text { cegueira congênita. }\end{array}$ & $\begin{array}{l}\text { Ficha de Avaliação física } \\
\text { específica e padronizada, } \\
\text { Ficha de Avaliação } \\
\text { psicológica também } \\
\text { específica, Ficha de } \\
\text { avaliação das atividades } \\
\text { das sessões. }\end{array}$ & $\begin{array}{l}\text { Melhoras em: aspectos motores } \\
\text { (marcha e equilíbrio), aspectos } \\
\text { cognitivos (aprendizagem de outros } \\
\text { sentidos), aspectos emocionais } \\
\text { (relações sociais e sentimento de } \\
\text { segurança), relacionamento social } \\
\text { (comunicação, atenção e regras } \\
\text { sociais) e na psicomotricidade } \\
\text { (equilíbrio e postura de tronco). }\end{array}$ \\
\hline $\begin{array}{l}\text { Marcelino, \& } \\
\text { Melo (2006) }\end{array}$ & $\begin{array}{l}\text { Duas crianças com } \\
\text { atraso de } \\
\text { desenvolvimento, } \\
\text { seus familiares e/ou } \\
\text { outros responsáveis } \\
\text { e profissionais da } \\
\text { instituição. }\end{array}$ & $\begin{array}{l}\text { Observação sistemática e } \\
\text { entrevistas } \\
\text { semiestruturadas. }\end{array}$ & $\begin{array}{l}\text { Há necessidade de: vinculação da } \\
\text { criança e da família com o animal e } \\
\text { com o processo terapêutico, interação } \\
\text { entre a criança, a equipe e outros } \\
\text { praticantes para socialização, e de } \\
\text { adequação dos estímulos do ambiente. } \\
\text { Melhora no aspecto socioafetivo da } \\
\text { criança, repercutindo nas relações } \\
\text { familiares. }\end{array}$ \\
\hline $\begin{array}{l}\text { Ávila et al. } \\
(2006)\end{array}$ & $\begin{array}{l}30 \text { crianças com } \\
\text { deficiências. }\end{array}$ & $\begin{array}{l}\text { Questionário aplicado às } \\
\text { mães desenvolvido para o } \\
\text { estudo. }\end{array}$ & $\begin{array}{l}96,6 \% \text { demonstrou melhoras com o } \\
\text { tratamento. Resultados em segurança } \\
(93,3 \%) \text {, mobilidade }(83,3 \%) \text {, atenção } \\
(76 \%) \text {, concentração }(46,6 \%) \text { e } \\
\text { afetividade }(56,6 \%) \text {. }\end{array}$ \\
\hline
\end{tabular}

\begin{tabular}{|c|c|c|c|}
\hline $\begin{array}{l}\text { Shultz et al. } \\
\text { (2007) }\end{array}$ & $\begin{array}{l}63 \text { crianças vítimas } \\
\text { de abuso ou } \\
\text { negligência com os } \\
\text { seguintes } \\
\text { transtornos: } \\
\text { Transtornos de } \\
\text { Humor, Transtorno } \\
\text { de Déficit de } \\
\text { Atenção e } \\
\text { Hiperatividade } \\
\text { (TDAH), Transtorno } \\
\text { do Estresse Pós- } \\
\text {-Traumático, } \\
\text { Transtorno de } \\
\text { ajustamento, } \\
\text { Transtorno } \\
\text { Disruptivo, e outros } \\
\text { transtornos. }\end{array}$ & $\begin{array}{l}\text { Children's Global } \\
\text { Assessment of } \\
\text { Functioning (GAF). }\end{array}$ & $\begin{array}{l}\text { Melhora na Global Assessment of } \\
\text { Functioning (GAF) em todas as } \\
\text { crianças ( } n=63) \text { e correlação entre o } \\
\text { percentual de melhora e o número de } \\
\text { sessões de equoterapia. Os sujeitos } \\
\text { mais novos apresentaram maiores } \\
\text { diferenças na GAF após intervenção. }\end{array}$ \\
\hline $\begin{array}{l}\text { Klontz et al. } \\
(2007)\end{array}$ & $\begin{array}{l}31 \text { participantes } \\
\text { adultos entre } 23 \text { e } \\
70 \text { anos de idade. }\end{array}$ & $\begin{array}{l}\text { Brief Symptom Inventory } \\
\text { (BSI); Personal } \\
\text { Orientation Inventory } \\
\text { (POI). }\end{array}$ & $\begin{array}{l}\text { Diminuição dos sintomas } \\
\text { psicopatológicos e aumento no bem- } \\
\text {-estar geral, por exemplo: maior foco } \\
\text { no presente e independência, } \\
\text { prontidão para vivenciar o aqui-agora, } \\
\text { menores índices de culpa, pesar e } \\
\text { medos relativos ao futuro. }\end{array}$ \\
\hline
\end{tabular}


Quadro I. Pesquisas recentes em equoterapia (continuação)

\begin{tabular}{|c|c|c|c|}
\hline Autor/ano & Participantes & Instrumentos & Resultados \\
\hline $\begin{array}{l}\text { Motti, \& Freire } \\
(2008)\end{array}$ & $\begin{array}{l}\text { Duas mulheres com } \\
\text { Transtorno de } \\
\text { Ansiedade } \\
\text { Generalizada (TAG). }\end{array}$ & $\begin{array}{l}\text { Ficha diária para avaliar as } \\
\text { sessões, Reasa (Roteiro } \\
\text { para Entrevista de } \\
\text { Avaliação dos Sintomas de } \\
\text { Ansiedade) elaborado } \\
\text { para o estudo e o relato } \\
\text { da psiquiatra que } \\
\text { acompanha os dois casos. }\end{array}$ & $\begin{array}{l}\text { Melhora em sintomas de ansiedade em } \\
\text { autoconfiança e segurança. }\end{array}$ \\
\hline $\begin{array}{l}\text { Davis et al. } \\
\text { (2009) }\end{array}$ & $\begin{array}{l}71 \text { crianças com } \\
\text { paralisia cerebral, } \\
\text { divididas em dois } \\
\text { grupos, } 35 \\
\text { praticantes de } \\
\text { equoterapia e } 36 \\
\text { controles. }\end{array}$ & $\begin{array}{l}\text { Gross Motor Function } \\
\text { Measure (GMFM); Child } \\
\text { Health Questionnaire; } \\
\text { Cerebral Palsy QoL-child } \\
\text { Kidscreen. }\end{array}$ & $\begin{array}{l}\text { Sem efeitos significativos após } 10 \\
\text { semanas, exceto na variável saúde da } \\
\text { família. }\end{array}$ \\
\hline $\begin{array}{l}\text { Keino et al. } \\
(2009)\end{array}$ & $\begin{array}{l}\text { Quatro crianças } \\
\text { com Transtorno do } \\
\text { Espectro do } \\
\text { Autismo (TEA). }\end{array}$ & $\begin{array}{l}\text { Escala Human-Equips- } \\
\text { Interaction on Mental } \\
\text { Activity Scale (HEIM). }\end{array}$ & $\begin{array}{l}\text { Melhora em comportamentos sociais e } \\
\text { de comunicação: maior contato visual } \\
\text { e adaptação às mudanças e menos } \\
\text { nervosismo e ansiedade. }\end{array}$ \\
\hline $\begin{array}{l}\text { Hameury et al. } \\
(2010)\end{array}$ & $\begin{array}{l}\text { Seis crianças com } \\
\text { TEA. }\end{array}$ & $\begin{array}{l}\text { Chelle d'Évaluation des } \\
\text { Comportements } \\
\text { Autistiques (ECA); grille } \\
\text { d'Évaluation Fonctionnelle } \\
\text { des Comportements } \\
\text { (EFC). }\end{array}$ & $\begin{array}{l}\text { Melhora em comunicação, imitação, } \\
\text { percepção, ajustamento motor e } \\
\text { emocional. }\end{array}$ \\
\hline $\begin{array}{l}\text { Memisevikj \& } \\
\text { Hodzhikj } \\
(2010)\end{array}$ & $\begin{array}{l}\text { Quatro crianças } \\
\text { com TEA entre } 8 \text { e } \\
10 \text { anos de idade. }\end{array}$ & $\begin{array}{l}\text { Autism Treatment } \\
\text { Evaluation Checklist } \\
\text { (ATEC). }\end{array}$ & $\begin{array}{l}\text { Melhoras significativas na comunicação, } \\
\text { socialização, integração sensorial e } \\
\text { cognitiva e no comportamento. }\end{array}$ \\
\hline $\begin{array}{l}\text { Favali, \& } \\
\text { Milton }(2010)\end{array}$ & $\begin{array}{l}\text { Cinco adultos (de } \\
36 \text { a } 66 \text { anos de } \\
\text { idade) com } \\
\text { esclerose múltipla, } \\
\text { acidente vascular } \\
\text { cerebral (AVC) e } \\
\text { paraplegia. }\end{array}$ & $\begin{array}{l}\text { Entrevista } \\
\text { semiestruturada com } 12 \\
\text { questões. }\end{array}$ & $\begin{array}{l}\text { Melhoras em quatro categorias: } \\
\text { benefícios físicos, benefícios } \\
\text { psicológicos, interação e } \\
\text { relacionamento com os animais, e } \\
\text { motivação e foco. }\end{array}$ \\
\hline $\begin{array}{l}\text { Prestes et al. } \\
(2010)\end{array}$ & $\begin{array}{l}\text { Duas crianças com } \\
\text { dificuldades de } \\
\text { aprendizagem. }\end{array}$ & $\begin{array}{l}\text { Escala de } \\
\text { Desenvolvimento Motor } \\
\text { (EDM) e Escala do Perfil } \\
\text { de Autopercepção para } \\
\text { Crianças (EPAPC). }\end{array}$ & $\begin{array}{l}\text { Melhoras em todas as áreas da EDM } \\
\text { dos dois participantes; na EPAPC, o } \\
\text { sujeito A evoluiu nos domínios do } \\
\text { Comportamento e Mais Valia Global; e } \\
\text { o sujeito B, na Competência Escolar e } \\
\text { Comportamento. }\end{array}$ \\
\hline $\begin{array}{l}\text { Cerino et al. } \\
(2011)\end{array}$ & $\begin{array}{l}24 \text { pacientes com } \\
\text { esquizofrenia; } \\
\text { desses, I } 4 \text { são } \\
\text { crônicos, todos } \\
\text { entre } 18 \text { e } 40 \text { anos. }\end{array}$ & $\begin{array}{l}\text { Brief Psychiatric Rating } \\
\text { Scale (BPRS) e 8-Items } \\
\text { Positive and Negative } \\
\text { Syndrome Scale (PANSS). }\end{array}$ & $\begin{array}{l}\text { Diminuição dos sintomas negativos e } \\
\text { positivos, principalmente apatia e } \\
\text { isolamento social nos dois grupos. }\end{array}$ \\
\hline
\end{tabular}




\section{Quadro I. Pesquisas recentes em equoterapia (continuação)}

\begin{tabular}{|c|c|c|c|}
\hline Autor/ano & Participantes & Instrumentos & Resultados \\
\hline $\begin{array}{l}\text { Holmes et al. } \\
(20 \mid 2)\end{array}$ & $\begin{array}{l}\text { II estudantes com } \\
\text { idades entre } 12 \text { e } 14 \\
\text { anos com } \\
\text { dificuldades } \\
\text { emocionais, } \\
\text { comportamentais ou } \\
\text { de aprendizagem. }\end{array}$ & $\begin{array}{l}\text { Escala de ansiedade } \\
\text { infantil de Spence (Spence } \\
\text { Children's Anxiety Scale - } \\
\text { SCAS); escala de } \\
\text { autoestima de Rosenberg } \\
\text { (Rosenberg Self-Esteem } \\
\text { Scale - SES); protocolo de } \\
\text { observação experimental } \\
\text { com duas dimensões: } \\
\text { aproximação e esquiva. }\end{array}$ & $\begin{array}{l}\text { Diminuição significativa em ansiedade } \\
\text { sem mudanças significativas em } \\
\text { autoestima após quatro sessões. } \\
\text { Manifestações de esquiva menores ao } \\
\text { final com maior diferença em relação } \\
\text { ao cavalo vivo do que em relação ao } \\
\text { cavalo de pau. }\end{array}$ \\
\hline
\end{tabular}

29 adolescentes entre 14 e 18 anos com dificuldades cognitivas, de ajustamento social, Bachi et al. de comunicação, de (20I2) controle de impulsos e de resolução de problemas divididos em dois grupos ( 15 sujeitos controles).

\section{Questionário de} autoimagem Offer SelfImage Questionnaire (OSIQ), questionário de comportamento de autocontrole para adolescentes Schedule for Assessing SelfControl Behaviors of Adolescents, escala de confiança interpessoal Children's Interpersonal

Trust Scale e escala de satisfação de vida para estudantes Student's Life Satisfaction Scale.
O estudo sugere tendência a mudanças positivas nos quatro parâmetros (autoimagem, autocontrole, confiança e satisfação com a vida) no grupo terapêutico, embora estatisticamente não tenham sido significativas.
Gabriels et al. (20I2)

\section{Aberrant Behavior} Checklist-community (ABC-C) para avaliar comportamentos de irritabilidade, estereotipias, letargia, hiperatividade e discurso inadequado; Vineland Adaptive Behavioral

42 crianças e adolescentes com TEA (36 meninos), entre 6 e 16 anos, 16 em grupo controle QI não verbal entre $44 \mathrm{e}$ 139; $38 \%$ com comorbidades.
Scales - Interview

Edition, Survey Form

(VABS-II) para verificar comportamentos adaptativos de comunicação, atividades de vida diária e habilidades de socialização; BruininksOseretsky Test of Motor Proficiency (BOT-2) para acessar habilidades motoras finas e grossas; e dois subtestes do Sensory Integration and Praxis Test (SIPT), Praxia para comandos verbais e Praxia postural.
Melhoras estatisticamente significativas em: Irritabilidade, letargia, comportamentos estereotipados, hiperatividade e discurso inadequado; melhoras no escore total de habilidades adaptativas e nos escores de comunicação, socialização e atividades diárias; melhoras nas habilidades motoras nas escalas de praxia verbal, praxia postural e de proficiência motora. 
Quadro I. Pesquisas recentes em equoterapia (continuação)

\begin{tabular}{|c|c|c|c|}
\hline Autor/ano & Participantes & Instrumentos & Resultados \\
\hline $\begin{array}{l}\text { Sunwoo et al. } \\
(20 \mid 2)\end{array}$ & $\begin{array}{l}\text { Oito pacientes (sete } \\
\text { homens) com } \\
\text { transtornos } \\
\text { neurológicos } \\
\text { crônicos e com } \\
\text { idades entre } 25 \text { e } 70 \\
\text { anos, média de } \\
\text { idade de } 42,4 \text { anos. }\end{array}$ & $\begin{array}{l}\text { Berg Balance Scale e } \\
\text { Tinetti Performance- } \\
\text { Oriented Mobility } \\
\text { Assessment, para avaliar } \\
\text { equilíbrio; IO Meter } \\
\text { Walking Test, para avaliar } \\
\text { a funcionalidade de } \\
\text { marcha; Modified Barthel } \\
\text { Index (K-MBI) para avaliar } \\
\text { atividade de vida diária; } \\
\text { Escala de Depressão de } \\
\text { Hamilton, Hamilton } \\
\text { Depression Rating Scale } \\
\text { (Ham-D) e Inventário } \\
\text { Beck de Depressão para } \\
\text { Coreanos - Korean Beck } \\
\text { Depression Inventory } \\
\text { (K-BDI), para avaliar o } \\
\text { humor. }\end{array}$ & $\begin{array}{l}\text { Melhoras em equilíbrio, aumento na } \\
\text { velocidade de marcha e nenhuma } \\
\text { alteração nas emoções e em atividades } \\
\text { de vida diária. }\end{array}$ \\
\hline $\begin{array}{l}\text { Jenkins, \& } \\
\text { Reed (20।3) }\end{array}$ & $\begin{array}{l}\text { Sete crianças e } \\
\text { adolescentes (seis } \\
\text { meninos) com TEA } \\
\text { entre seis e } 14 \text { anos } \\
\text { de idade (média de } \\
\text { idade de } 9,5 \text { anos) } \\
\text { divididos em dois } \\
\text { grupos. }\end{array}$ & $\begin{array}{l}\text { Situação estruturada de } \\
\text { observação e Escala de } \\
\text { comportamentos infantis } \\
\text { preenchida pelos pais } \\
\text { Child Behavior Checklist } \\
\text { (CBCL). }\end{array}$ & $\begin{array}{l}\text { Não houve melhoras nos } \\
\text { comportamentos de iniciativa e de } \\
\text { comandos vocais. Houve melhora na } \\
\text { postura sobre o cavalo, aumento de } \\
\text { comandos não verbais (gestos), maior } \\
\text { aceitação às regras (conformidade), } \\
\text { menos problemas de } \\
\text { comportamentos, porém poucos } \\
\text { resultados foram significativos. }\end{array}$ \\
\hline $\begin{array}{l}\text { Lanning et al. } \\
(20 \mid 4)\end{array}$ & $\begin{array}{l}25 \text { crianças e } \\
\text { adolescentes com } \\
\text { TEA entre quatro e } \\
15 \text { anos de idade, } \\
\text { divididas em dois } \\
\text { grupos ( } 12 \\
\text { controles). }\end{array}$ & $\begin{array}{l}\text { Escala de Qualidade de } \\
\text { vida para crianças } \\
\text { Pediatric Quality of Life } \\
4.0 \text { Generic Core Scales } \\
\text { (PedsQL); e Escala infantil } \\
\text { de qualidade de vida para } \\
\text { condições de saúde } \\
\text { crônicas e agudas Child } \\
\text { Health Questionnaire } \\
\text { (CHQ). }\end{array}$ & $\begin{array}{l}\text { Melhoras em qualidade de vida de } \\
\text { praticantes de equoterapia foram } \\
\text { superiores quando comparado ao } \\
\text { grupo controle. Maiores efeitos } \\
\text { positivos em socialização, } \\
\text { funcionalidade física, funcionalidade } \\
\text { escolar e saúde mental e } \\
\text { comportamental geral. Os ganhos em } \\
\text { funcionalidade emocional, social e física } \\
\text { foram significativos após } 6 \text { semanas de } \\
\text { tratamento depois se estabilizou. }\end{array}$ \\
\hline $\begin{array}{l}\text { Rosario- } \\
\text { Montejo et al. } \\
(2014)\end{array}$ & $\begin{array}{l}\text { II crianças e } \\
\text { adolescentes com } \\
\text { déficit em } \\
\text { desenvolvimento } \\
\text { psicomotor leve } \\
\text { entre três e I5 anos } \\
\text { de idade, seis } \\
\text { meninos. }\end{array}$ & $\begin{array}{l}\text { Gross Motor Function } \\
\text { Measure (GMFM-88) } \\
\text { como medida de } \\
\text { funcionalidade motora e } \\
\text { Pediatric Quality of Life } \\
\text { Inventory (PedsQL) para } \\
\text { avaliar a qualidade de } \\
\text { vida. }\end{array}$ & $\begin{array}{l}\text { Houve diferença significativa antes e } \\
\text { depois somente na função motora } \\
\text { (GMFM-88). Apesar do aumento dos } \\
\text { escores em qualidade de vida, esta não } \\
\text { foi estatisticamente significativa. }\end{array}$ \\
\hline
\end{tabular}

Elaborado pelas autoras. 


\section{Discussão}

Dentre os 20 estudos revisados, houve predominância do uso de escalas de qualidade de vida na avaliação dos praticantes, mesmo que algumas destas não sejam as melhores para avaliar pessoas com deficiências físicas, escopo predominante na equoterapia. Os efeitos observados após o tratamento por vezes não foram estatisticamente significativos e alguns autores dos estudos revisados creditam tal achado ao tipo de parâmetro escolhido para avaliar a intervenção. Por exemplo, a escala pediátrica de qualidade de vida apresentou pouca sensibilidade para avaliar mudanças em crianças com paralisia cerebral nos estudos de Davis et al. (2009) e de Rosario-Montejo et al. (2014), divergindo dos resultados da pesquisa de Lanning et al. (2014) que usou outra versão para avaliar as crianças, Pediatric Quality of Life 4.0 Generic Core Scales (PedsQL).

Apesar de encontrar melhoras em saúde da família, avaliada pelo questionário de saúde após 10 semanas de tratamento, pudemos verificar que não houve efeitos significativos em habilidades motoras amplas, no estudo com maior número de participantes (71 crianças com paralisia cerebral) (Davis et al., 2009). Talvez esse resultado possa estar relacionado aos atendimentos realizados às famílias que são atendidas em equoterapia. Segundo levantamento brasileiro, $43,8 \%$ dos psicólogos referiram atender frequentemente as famílias (Zamo, 2016).

Outros estudos de revisão sistemática em equoterapia também apontam maior número de investigações sobre benefícios físicos, como equilíbrio, amplitude de movimento e qualidade de marcha (Smith-Osborne \& Selby, 2010; Sterba, 2007). Assim como nosso estudo, Kendall et al. (2015) se detiveram em verificar os efeitos sobre aspectos emocionais, cognitivos e sociais da equoterapia em pesquisas publicadas.

Os benefícios da equoterapia nas relações sociais e no sentimento de segurança foi evidenciado em nossa revisão e na de Kendall et al. (2015). Como optamos por selecionar artigos em português, espanhol, inglês e francês, conseguimos analisar maior quantidade de estudos, sendo este um dos avanços de nossa revisão em comparação aos estudos que se limitam à língua inglesa. Essa inclusão, no entanto, possibilita críticas às pesquisas no Brasil que necessitam de maior rigor metodológico e utilização de instrumentos de mensuração internacionalmente reconhecidos, não sendo exclusividade brasileira a necessidade de rigor metodológico nas pesquisas em equoterapia quando os avaliamos nos parâmetros de evidências em saúde (Brasil, 2014) .

Para fazermos comparação entre os estudos, seria necessária maior homogeneidade de variáveis, grupos clínicos mais bem definidos, instrumentos com validade transcultural e procedimentos terapêuticos uniformizados em número, frequência e tempo de sessão. O que Schulz et al. (2007) comprovaram foi que há correlação positiva entre o percentual de melhora e o número de sessões de equoterapia, e quando realizada em crianças mais jovens essas apresentam melhores resultados na GAF quando comparadas com as mais velhas, também vítimas de abuso ou negligência. Ainda não há nessa área um guideline ou protocolo de intervenção bem definido segundo os objetivos terapêuticos. 
A falta de evidências empíricas em equoterapia é um desafio a quem pretende validar este método terapêutico. Anestis, Anestis, Zawilinski, Hopkins, \& Lilienfeld (2014) salientam este empecilho nos recortes de artigos com enfoque nos transtornos mentais. Além da falta de dados empíricos, verificamos falhas metodológicas, pequeno número de participantes e conclusões que extrapolam a capacidade dos dados apresentados. Uma das formas de minimizar esta lacuna poderia ser o emprego da avaliação psicológica com testes psicológicos validados e fidedignos como um parâmetro inicial e pós intervenção. Mesmo que as escalas de autorrelato que sejam passíveis de vieses de resposta, são mais confiáveis do que entrevistas semiestruturadas conduzidas pelos próprios pesquisadores, pois proporcionam maior capacidade de replicação de estudos (Ziegler, 2015).

\section{Considerações finais}

Por meio deste estudo de revisão sistemática pudemos verificar que a maioria dos estudos no âmbito psicológico foi realizado com crianças e adolescentes, e principalmente com aquelas com diagnóstico de TEA. Talvez a realização de estudos com controle de variáveis intervenientes ou metodologias com grupo controle pudessem evidenciar mais claramente os efeitos da equoterapia. Os estudos de caso são importantes para aprofundar de modo particular com pouca capacidade de generalização. Os parâmetros ideais de evidências em saúde sugerem o uso de medidas com menor viés, e com parâmetros iniciais e finais claros na avaliação da eficácia (Brasil, 2014). Há uma diversa gama de instrumentos de avaliação psicológica que poderiam servir a este propósito, porém nos estudos revisados não evidenciamos o uso de muitos instrumentos de avaliação psicológica.

Como limitações deste estudo salientamos as dificuldades encontradas para definir o que seria objeto de estudo da psicologia. Com receio de deixar alguns artigos fora de nossa revisão devido à falta de clareza nos parâmetros avaliados, incluímos artigos sobre funcionalidade e psicomotricidade além de aspectos emocionais e cognitivos. Havia um estudo em japonês que não conseguimos uma versão em inglês, portanto essa pesquisa ficou fora da revisão. Apesar disso, os estudos revisados apontaram resultados positivos desta intervenção em socialização e comunicação, principalmente.

Ainda assim, salientamos que os resultados de alguns estudos não são claros quanto à eficácia do método terapêutico da equoterapia. Por conseguinte, parâmetros utilizados para avaliar em consultório podem não ser úteis na avaliação no setting da equoterapia. Há características do picadeiro e da interação entre praticante e cavalo que são demandas da atividade equestre e pouco replicáveis no consultório. Desse modo, o desenvolvimento de instrumentos com validade ecológica para o setting da equoterapia (avaliação sobre o cavalo) seria muito importante. Apesar deste ideal, alguma avaliação psicológica prévia à atividade de equoterapia já seria importante no desenvolvimento científico da área. Mesmo que fatores intervenientes não possam ser controlados ao longo da intervenção, uma bateria de avaliação ao ser reaplicada no 
praticante forneceria mais dados a serem agregados aos relatórios de observação dos profissionais da psicologia que atuam na equoterapia.

\section{Referências}

Associação Nacional de Equoterapia (Ande-BRASIL) (2016). Disponível em: http:// equoterapia.org.br/articles/index/article_detail/142/2022.

Anestis, M., Anestis, J., Zawilinski, L., Hopkins, T., \& Lilienfeld, S. (2014). Equinerelated treatments for mental disorders lack empirical support: a systematic review of empirical investigations. Journal of Clinical Psychology, 70(12), 11151132. DOI: $10.1002 / j c l p .22113$.

Ávila, A., Brito, Y., Pupo, F., \& Roselló, A. (2006). Equinoterapia: experiencia en un año de tratamiento. Archivo Médico de Camagüey, 10(6), 1-5.

Bachi, K., Terkel, J. \& Teichman, M. (2012). Equine-facilitated psychotherapy for at-risk adolescents: the influence on self-image, self-control and trust. Clinical Child Psychology and Psychiatry, 17(2), 298-312. DOI: 10.1177/1359104511404177.

Câmara dos Deputados. (2016). Câmara regulamenta uso da equoterapia na reabilitação de pessoas com deficiência. Disponível em: http://www2.camara.leg.br/ camaranoticias/noticias/SAUDE/491554-CAMARA-REGULAMENTA-USO-DA-EQUOTERAPIA-NA-REABILITACAO-DE-PESSOAS-COM-DEFICIENCIA.html.

Cerino, S., Cirulli, F., Chiarotti, F., \& Seripa, S. (2011). Non conventional psychiatric rehabilitation in schizophrenia using therapeutic riding: the FISE multicentre Pindar Project. Annali dell'lstituto Superiore di Sanità, 47(4), 409-414. DOI: 10.4415/ANN_11_04_13.

Cruz, V. (2010). A psicologia na equoterapia: dúvidas e certezas. Revista Brasileira de Equoterapia, 21(22), 18-21.

Davis, E., Davies, B., Wolfe, R., Raadsveld, R., Heine, B., Thomason, P., Dobson, F., \& Graham, H. (2009). A randomized controlled Trial of the impact of therapeutic horse riding on the quality of life, health, and function of children with cerebral palsy. Developmental Medicine \& Child Neurology, 51(2), 111-119. DOI: 10.1111/j.1469-8749.2008.03245.x.

Favali, V., \& Milton, M. (2010). Disabled horse-rider's experience of horse-riding: exploring the therapeutic benefits of contact with animals. Existential Analysis, $21(2), 251-262$.

Freire, H. (1999). Equoterapia Teoria e prática: uma experiência com crianças autistas. São Paulo: Vetor. 
Gabriels, R., Agnew, J., Holt, K., Shoffner, A., Zhaoxing, P., Ruzzano, S., Clayton, G., \& Mesibov, G. (2012). Pilot Study mesuring the effects of therapeutic horseback riding on school-age children and adolescents with autism spectrum disorders. Research in Autism Spectrum Disorders, 6(2), 578-588. DOI: 10.1016/j. rasd.2011.09.007.

Giagazoglou, P., Arabatzi, F., Dipla, K., Liga, M., \& Kellis, E. (2012). Effect of a hippotherapy intervention program on static balance and strength in adolescents with intellectual disabilities. Research in Developmental Disabilities, 33(6), 22652270. DOI: 10.1016/j.ridd.2012.07.004.

Hameury, L., Delavous, P., Teste, B., Leroy, C., Gaboriau, J.-C., \& Berthier, A. (2010). Équithérapie et autisme. Annales Médico-Psychologiques, 168(9), 655-659. DOI: 10.1016/j.amp.2009.12.019.

Holmes, C., Goodwin, D., Redhead, E. \& Goymour, K. (2012). The Benefits of Equine-Assisted Activities: An Exploratory Study. Child and Adolescent Social Work Journal, 29(2), 111-122. DOI: 10.1007/s10560-011-0251-z.

Jenkins, S., \& Reed, F. (2013). An experimental analysis of the effects of therapeutic horseback riding on the behavior of children with autism. Research in Autism Spectrum Disorders, 7(6), 721-740. DOI: 10.1016/j.rasd.2013.02.008.

Keino, H., Funahashi, A., Keino, H, Miwa, C., Hosokawa, M., Hayashi, Y., \& Kawakita, K. (2009). Psycho-educational horseback riding to facilitate communication ability of children with pervasive developmental disorders. Journal of Equine Science, 20(4), 79-88. DOI: 10.1294/jes.20.79.

Kendall, E., Maujean, A., Pepping, C., Downes, M., Lakhani, A., Byrne, J., \& Macfarlane, K. (2015). A systematic review of the efficacy of equine-assisted interventions on psychological outcomes. European Journal of Psychotherapy \& Counselling, 17(1), 57-79. DOI: 10.1080/13642537.2014.996169.

Klontz, B., Bivens, A., Leinart, D., \& Klontz, T. (2007). The effectiveness of equineassisted experiential: results of an open clinical trial. Society and Animals, 15(3), 257-267. DOI: 10.1163/156853007X217195.

Lanning, B., Baier, M., Ivey-Hatz, J., Krenek, N., \& Tubbs, J. (2014). Effects of equine assisted activities on autism spectrum disorder. Journal of Autism and Developmental Disorders, 44(8), 1897-1907. DOI: 10.1007/s10803-014-2062-5.

Malta, S. (2002). A visão da Psicologia na equoterapia: o uso do cavalo como instrumento facilitador. In Associação Nacional de Equoterapia. (Eds.) Coletânea de Trabalhos, II Congresso Brasileiro de Equoterapia. Jaguariúna: Ande/BRASIL.

Marcelino, J., \& Melo, Z. (2006). Equoterapia: suas repercussões nas relações familiares da criança com atraso de desenvolvimento por prematuridade. Estudos de Psicologia de Campinas, 23(3), 279-287. 
Medeiros, M., \& Dias, E. (2002). Equoterapia: bases e fundamentos. Rio de Janeiro: Revinter.

Memisevikj, X., \& Hodzhikj, S. (2010). The effects of equine-assisted therapy in improving the psycho-social functioning of children with autismo. Journal of Special Education and Rehabilitation, 11(3-4), 57-67.

Ministério da Saúde. (2014). Diretrizes metodológicas Sistema GRADE - Manual de Graduação de Qualidade da Evidência e Força de Recomendação para Tomada de Decisão em Saúde. Brasília, DF, Brasil. Disponível em: <www.saude.gov;br/bvs>.

Ministério do Trabalho e Emprego. (2016). Classificação Brasileira das Ocupações. Disponível em: http://www.mtecbo.gov.br/cbosite/pages/home.jsf.

Motti, G., \& Freire, H. (2008). A prática da equoterapia como tratamento para pessoas com ansiedade. In Associação Nacional de Equoterapia (Eds.). Coletânea de Trabalhos do IV Congresso Brasileiro de Equoterapia - I Congresso Latino-Americano de Equoterapia (pp. 151-159). Curitiba, PR.

Nunes, M., Muniz, M., Reppold, C., Faiad, C., Bueno, J. \& Noronha, A. (2012). Diretrizes para o ensino de avaliação psicológica. Instituto Brasileiro de Avaliação Psicológica. Disponível em: http://www.ibapnet.org.br/docs/ensino_de_avaliacao_psicologica.pdf.

PATH. (2016). Professional Association of Therapeutic Horsemanship International. Disponível em: http://www.pathintl.org/.

Pauw, J. (2000). Therapeutic horseback riding studies: problems experienced by researchers. Physiotherapy, 86(10), 523-527.

Prestes, D., Weiss, S., \& Araújo, J. (2010). A equoterapia no desenvolvimento motor e autopercepção de escolares com dificuldade de aprendizagem. Ciências \& Cognição, 15(3), 192-203.

Rosario-Montejo, O., Molina-Rueda, F., Muñoz-Lasa, S., \& Alguacil-Diego, I. (2014). Efectividad de la terapia ecuestre en niños con retraso psicomotor. Neurología, 30(7), 425-432. DOI: 10.1016/j.nrl.2013.12.023.

Schultz, P., Remick-Barlow, A., \& Robbins, L. (2007). Equine-assisted psychotherapy: a mental health promotion/intervention modality for children who have experienced intra-family violence. Health and Social Care in the Community, 15(3), 265-271. DOI: 10.1111/j.1365-2524.2006.00684.x.

Senado Federal. (2016). Substitutivo da Câmara dos Deputados, $n^{\circ} 13$, de 2015, ao Projeto de Lei do Senado, $\mathrm{n}^{\circ} 264$, de 2010. Disponível em: http://www25.senado. leg.br/web/atividade/materias/-/materia/122814.

Shurtleff, T., Standeven, J., \& Engsberg, J. (2009). Changes in dynamic trunk/head stability and functional reach after hippotherapy. Archives of Physical Medical Rehabilitation, 90(7), 1185-1195. DOI: 10.1016/j.apmr.2009.01.026. 
Silva, C. (2003). Equoterapia para cegos: efeitos e técnica de atendimento. (Dissertação de Mestrado). Universidade Católica Dom Bosco, Campo Grande, MS, Brasil.

Silva, C., \& Grubits, S. (2004). Discussão sobre o efeito positivo da equoterapia em crianças cegas. PSIC - Revista de Psicologia da Vetor Editora, 5(2), 6-13.

Smith-Osborne, A., \& Selby, A. (2010). Implications of the literature on equine-assisted activities for use as a complementary intervention in social work practice with children and adolescents. Child Adolescence Social Work Journal, 27(4), 291-307. DOI: 10.1007/s10560-010-0201-1.

Sterba, J. (2007). Does horseback riding therapy or therapist-directed hippotherapy rehabilitate children with cerebral palsy? Developmental Medicine \& Child Neurology, 49(1), 68-73. DOI: 10.1017/S0012162207000175.

Sunwoo, H., Chang, W., Kwon, J., Kim, T., Lee, J., \& Kim, Y. (2012). Hippotherapy in adult patients with chronic brain disorders: a pilot study. Annals of Rehabilitation Medicine, 36(6), 756-761. DOI: 10.5535/arm.2012.36.6.756.

Zamo, R. (2016). Construção de uma bateria infantil de avaliação neuropsicológica para atividade equestre. Tese de Doutorado, Universidade Federal do Rio Grande do Sul, Porto Alegre, RS, Brasil.

Ziegler, M. (2015). "F*** You, I Won't Do What You Told Me!" - Response biases as threats to psychological assessment. European Journal of Psychological Assessment, 31(3), 153-158. DOI: 10.1027/1015-5759/a000292.

Submissão: 12.1.2016

Aceitação: 26.9.2016 